\title{
Flow structure and mixing dynamics of the advected line thermal
}

GASKIN, S.J., Assistant Professor, Dept. Civil Engineering and Applied Mechanics, McGill University, Montreal Canada, H2A 2 K6.

WOOD, I.R., Emeritus Professor, Department of Civil Engineering, University of

Canterbury, Christchurch, New Zealand.

\begin{abstract}
Ocean outfalls are modelled as buoyant jets. The final behaviour in a deep ocean is the advected line thermal, which is dominated by the jet buoyancy and the ambient velocity. The dilution of the effluent along its path is of interest and in some cases the traditional time averaged information is needed and in others the instantaneous or peak concentrations are needed. Laser induced fluorescence (LIF) is used to study the instantaneous flow structure and the mixing dynamics of the advected line thermal. Large scale structures in the flow are identified as subthermals and their development influences the flow behaviour. Statistical measures of the magnitude of the concentration fluctuations and the intermittency of the flow are obtained from LIF images.
\end{abstract}

\section{Introduction}

A major concern in environmental hydraulics is the fate and transport of pollutants released into surface water bodies in terms of the dilution of the effluent along its path. The dilution minimizes the detrimental effects on the environment. Effluent releases are often buoyant due to the density difference between the effluent and the receiving fluid and the receiving fluid may have a current. Examples include hot gases released from a chimney stack in a wind, sewage released into the ocean and being advected by the ocean current and cooling water plumes released into lakes and rivers.

The effluent releases are modelled as buoyant jets in a cross-flow, whose behaviour can be divided into several asymptotic regions in which different parameters dominate. The behaviour progresses from a momentum dominated jet, to a buoyancy dominated plume, and finally to a buoyancy and crossflow dominated advected line thermal as shown in Fig.1. It is this advected line thermal or bent-over plume region that is the subject of this paper.

Previous studies have investigated the time averaged behaviour of the flow in terms of the 
average concentration decay, width and trajectory. For the asymptotic flow regions this can be determined by simple dimensional analysis and for the transition between these region an integral analysis, based on a Eulerian (Wood et al. [14]) or a lagrangian approach (Lee and Cheung [4]), can be used.

Experiments have shown that the time averaged structure of the advected line thermal is a counter rotating vortex pair. Morton, Nguyen and Cresswell ([1], [5], [6]) after reviewing past work on line thermals (Scorer [12], Richards [11] and Tsang [13]) proposed that the variability between and within the experimental results was due to the inherent unsteadiness of the line thermal. This is confirmed by looking at the instantaneous flow, which has a highly variable and complex 3-dimensional structure. Knowledge of the 3-D structure is important in understanding the mixing dynamics of the flow and in determining peak concentrations, which are important in applications where short term toxic concentrations of pollutants or self-ignitable concentrations of combustion mixtures are present.

In this study the instantaneous structure of the advected line thermal was investigated using laser induced fluorescence to obtain details of the variations of the concentration in space, time and intensity. Large scale structures in the flow were identified as subthermals and their development followed. Statistical measures of the flow structure were obtained in terms of the magnitude of the concentration fluctuations and the intermittency of the flow.

\section{Experimental Method}

The advected line thermal was modelled by releasing a negatively buoyant $3 \%$ salt solution at a velocity of $U_{0}$ from a horizontal port into a glass walled tank $(6 \times 1.5 \times 1 \mathrm{~m})$. To model the crossflow the port was towed through the tank at a velocity of $\mathrm{U}_{\infty}$ where $\mathrm{U}_{0}=\mathrm{U}_{\infty}$. Qualitative and quantitative details of the flow geometry and concentration were obtained with a planar laser induced fluorescence system. The system used a $2.5 \mathrm{~W}$ argon ion laser combined with a rotating and a parabolic mirror to create a collimated laser sheet and the salt solution was dyed with a fluorescent dye Rhodamine 6G. At the low concentrations used, the Rhodamine 6G fluoresces when excited by the laser sheet with an intensity which varies linearly with the amount of dye present allowing flow concentrations to be determined (Gaskin [2]). The laser sheet was oriented vertically and placed so that both longitudinal centreline sections and cross-sections of the flow were obtained.

A CCD camera recorded the data in digital light intensity images with an area resolution per pixel 
of $0.681_{P, A}$ wide by $0.731_{P, A}$ high $\left(l_{P, A}=0.00153 \mathrm{~m}\right)$, a laser sheet thickness of $2.01_{P, A}$, and a temporal resolution of $0.04 \mathrm{~s}$. For the quantitative analysis a number of profiles were investigated at distances from the port of $\mathrm{x} / \mathrm{l}_{\mathrm{P}, \mathrm{A}}=141,285,429,573,785$, and 976 . The data for each profile came from 3 different experiments, with approximately 20 profiles taken from each experiment for a total of 60 instantaneous profiles at each location. The profile data was normalised by the maximum concentration, width and midpoint of the width of the time averaged profile.

The initial parameters of the 6 experimental advected line thermals used in the study were as follows : $\mathrm{d}_{\mathrm{p}}=0.0104 \mathrm{~m}, \mathrm{U}_{0}=\mathrm{U}_{\infty}=0.11 \mathrm{~m} / \mathrm{s}, \mathrm{C}_{0}=30 \mathrm{~g} / 1 \mathrm{salt}, \Delta_{0}=0.218 \mathrm{~m} / \mathrm{s}^{2}$, $\mathrm{q}_{\Delta 0}=2.04 \times 10^{-6} \mathrm{~m}^{4} / \mathrm{s}^{3}, 1_{\mathrm{P}, \mathrm{A}}=0.00153 \mathrm{~m}, \mathrm{Fr}=2.31$, and $\mathrm{R}_{\mathrm{e}} .1100$, all values are $\pm 3-5 \%$.

\section{Flow structure of Advected Line Thermal}

The time averaged view of the advected line thermal shows a counter rotating vortex pair in the cross-section and a regular concentration decay with distance in the longitudinal centreline section shown in Fig. 2. However, the laser induced fluorescence technique, which enables fine details of the flow structure to be seen, shows that the time averaged view is a simplification of the complex and irregular flow seen in instantaneous images. This is illustrated in Fig. 3 and Fig. 4, which show the development of a section of the advected line thermal as it falls through the ambient and is advected away from the port at the crossflow velocity.

The flow initially bears some resemblance to the two dimensional vortex pair or line thermal model but as the distance from the port increases the structure breaks up and becomes irregular. In the later stages of development, the structure is dominated by the formation of 3-D subthermal structures protruding from the main flow structure. The general structure of a line thermal and a 3-D thermal are sketched in Fig.5. The flow just after release, the significant stages in the flow development and the development of the subthermal structures are explained in more detail below.

Initial release: The structured flow pattern, shown in Fig. 6, is initiated from the vorticity shed from the port exit. This initial flow structure is regular and similar to the coherent structures seen in the flow of smoke from a glass tube by Perry and Lim [10]. They suggest that the structure might be relevant to the large-scale motions in fully turbulent flow. In the advected thermal flow, the subsequent development of the subthermal structures is determined in part by the initial 
disturbances of the linear distribution of the buoyant fluid (i.e. formation of the structures at the port exit).

Flow development: (observations from longitudinal (L) and cross-sectional (x) views, Fig. 2 and Fig.3) $0-1301_{\mathrm{PA}}:$ (L) The initial release structures form and the flow is fairly coherent. (x) The concentration distribution develops from the initially Gaussian to a vortex pair distribution as the downward motion of the buoyant fluid begins.

$130-2601_{\mathrm{PA}}:$ (L) The initial structures either merge and grow or disappear as their fluid is drawn into adjacent structures, see Fig. 7. The negatively buoyant fluid begins to concentrate in the lower half of the flow region with high density gradients existing at the leading edge. (x) The vortex pair shape develops but begins break up and be non-contiguous.

$260-1000 \mathrm{l}_{\mathrm{PA}}$ : (L) The irregularity of the flow increases as subthermals form and fall away from main flow region. (x) The cross-sections can now be roughly categorised as being at subthermals or between subthermals. Between subthermals, Fig. 4a, cross-sections are now a non-contiguous horseshoe shape in which the dominant motion is that of a counter rotating vortex pair. At the subthermals, which contain most of the buoyancy, cross-sections are a Yshape, with the 'leg' being the subthermal and the 'arms' being the remains of the vortex pair structure, see Fig. 4b.

$>10001_{\mathrm{PA}}$ : (L) Subthermals structures contain most of the buoyant fluid and are spaced at quasi regular intervals.

Subthermal structures: Subthermal structures occur because disturbances at release cause the buoyant fluid to become unevenly distributed along the advected thermal. The leading edge of the advected thermal is hydrodynamically unstable so any initial disturbance increase in size with time. Regions of the flow, in which there is relatively more buoyant fluid, fall faster due to their greater buoyant force and protrude from the main flow as subthermals. As shown in Fig. 8, the subthermals are initially similar to an axisymmetric thermal, forming a cap, within which is a vortex ring, and entraining fluid at their rear. The subthermals are connected back to the main flow region by a stem of buoyant fluid. As the buoyant fluid is carried downwards in the subthermal structures, buoyant fluid from the main region starts to flow towards the subthermals, as shown in Fig. 7.

In later stages of the subthermal development successive cap and vortex ring structures form. As the first cap and vortex ring falls it becomes diluted and the fluid drawn in behind it is then denser so it falls through the centre of the old cap forming a new cap and vortex ring. This can 
repeat a number of times as illustrated in Fig. 9.

\section{Concentration Fluctuations}

The instantaneous view of the advected line thermal does not have the smooth concentration gradients seen in the time-averaged data due to its turbulent nature and the developing subthermal structures. The subthermal development entrains fluid into the flow resulting in regions of relatively high concentration and other regions of relatively low concentration. These regions fluctuate in size and concentration as the flow develops, resulting in relatively large concentration fluctuations throughout the flow.

The concentration fluctuations are quantified along profiles across the flow primarily using the root mean squared deviations between the instantaneous and the averaged concentrations, which are normalised by the maximum of the averaged profile, $\mathrm{rms} \mathrm{c}^{\prime} / \overline{\mathrm{C}}_{\max }$, given by

$$
\operatorname{rms} \frac{\mathrm{c}^{\prime}}{\overline{\mathrm{C}}_{\max }}=\frac{\sqrt{\frac{1}{\mathrm{n}} \sum_{1}^{\mathrm{n}} \mathrm{c}^{\prime 2}}}{\overline{\mathrm{C}}_{\max }}
$$

where $\mathrm{c}^{\prime}$ is the concentration fluctuation at a point equal to $* \mathrm{c}_{\text {instantaneous }}-\mathrm{c}_{\text {averaged }} *, \overline{\mathrm{C}}_{\max }$ is the maximum of the average profile and $\mathrm{n}$ is the number of data points. Further information about the flow is obtained by comparing the shape of the $\mathrm{rms} \mathrm{c}^{\prime} / \overline{\mathrm{C}}_{\max }$ to the shape of the averaged profile and to the maximum concentration values along the profile.

The instantaneous profiles illustrate how the flow structure becomes more irregular further from the port due to the development of the subthermal structures and the turbulent nature of the flow as seen in Fig.10. The instantaneous profiles closest to the port are single peaked but further from the port the profiles have multiple peaks, although averaged over time all profiles had an approximately Gaussian profile.

The extreme values to which the concentrations fluctuate are investigated through the maximum and minimum instantaneous concentrations that are found for every point in each of the investigated profiles. The minimum concentrations are zero along each profile indicating that ambient fluid can be present at any location in the flow as a result of entrainment and the development of the subthermals. The peak or maximum concentration profiles plotted in Fig. 11, are self-similar in shape and skewed with higher maximum concentrations, of up to 3.5 to 4 times the maximum of the averaged data. The maximum concentrations found depend on the temporal and spatial resolution of the data as coarser resolutions will result in some averaging of 
peak values. The current data with a measurement volume of $11_{\mathrm{P}, \mathrm{A}}^{3}$ will slightly underestimate the true maximum. Maximum concentrations are found near the leading edge because fluid with higher concentrations, i.e. a greater buoyancy deficit, falls initially to the leading edge of the flow, then falls away from the leading edge as it develops into a subthermal. An abrupt increase in maximum concentration occurs at $\mathrm{r} / \mathrm{b}_{\mathrm{V}}=1.5$ to 2 . The average concentration profiles in Fig. 10 show that the non-zero concentrations start at $r / b_{v}=2$. The abrupt increase indicates the high concentration gradients at the leading edge of the thermal. At the trailing edge, maximum concentrations of approximately $0.5 \overline{\mathrm{C}}_{\max }$ (of the averaged profile) from $\mathrm{r} / \mathrm{b}_{\mathrm{V}}=-2$ onwards occur, which indicate the presence of buoyant fluid that does not get entrained into the downward moving vortex structures but is left in a sort of wake stretched out behind the main advected thermal flow.

Profiles of rms c'/ $\overline{\mathrm{C}}_{\max }$ along the advected thermal, plotted in Fig. 11, quantify the concentration fluctuations. The profiles are self-similar and show a skewed distribution with $\mathrm{rms} \mathrm{c}^{\prime} / \overline{\mathrm{C}}_{\max }$ increasing towards the leading edge then dropping off rapidly indicating an asymmetric flow. The maximum $\mathrm{rms} \mathrm{c} / \overline{\mathrm{C}}_{\max }$ of 0.8 occurs towards the leading edge of the flow at $\mathrm{r} / \mathrm{b}_{\mathrm{v}}=0.75$ to 1.0. An abrupt (although somewhat variable) increase in $\mathrm{rms} \mathrm{c} / \overline{\mathrm{C}}_{\max }$ occurs at a distance of between $\mathrm{r} / \mathrm{b}_{\mathrm{V}}=1.5$ to 2 , again corresponding to the high concentration gradients at the leading edge of the advected thermal and its subthermals. The variability in the abrupt increase of rms $c^{\prime} / \overline{\mathrm{C}}_{\max }$ is due to the averaging out of the different distances to which the subthermals extend downwards at a given distance from the port. At the trailing edge, the decrease in $\mathrm{rms} \mathrm{c}^{\prime} / \overline{\mathrm{C}}_{\max }$ is more gradual and tails off to a value of approximately $0.1 \mathrm{at} r / \mathrm{b}_{\mathrm{V}}=-2.0$. These non-zero values of $\mathrm{rms} \mathrm{c} / \overline{\mathrm{C}}_{\max }$ again indicate the presence of small amounts of buoyant fluid that get left behind by the main downward movement of the advected thermal.

The rms $\mathrm{c}^{\prime} / \overline{\mathrm{C}}_{\max }$ profile shape differs from the maximum concentration profiles in that there is a fairly linear decrease in variability from the leading edge to the trailing edge, whereas the highest local maximum concentrations can be found anywhere from the leading edge to an $r / b_{\mathrm{V}}$ of -0.5 . This indicates that on average the concentration is higher about the centreline although the peak concentrations can be found both in the centreline area and up to the leading edge of the flow. The mixing dynamics of the advected line thermal differ from those of the jet and the plume, which is indicated by the asymmetry of the flow and the larger $c^{\prime} / \overline{\mathrm{C}}_{\max }$ values. Kotsovinos [3] found, for the plane jet-like flows, the $\mathrm{rms} \mathrm{c}^{\prime} / \overline{\mathrm{C}}_{\max }$ was self-similar with a double peaked shape and increased in magnitude with distance along the jet. In the plane plumes, the $\mathrm{rms} \mathrm{c}^{\prime} / \overline{\mathrm{C}}_{\max }$ was 
self-similar with a top hat shape having a peak value of 0.40. Papaniculaou [7] studied axisymmetric jets and plumes. The rms $\mathrm{c}^{\prime} / \overline{\mathrm{C}}_{\max }$ profiles were self-similar when scaled by the width and the maximum concentration of the average data. The axisymmetric jets had a double peaked profile with peak values of $\mathrm{rms} \mathrm{c}^{\prime} / \overline{\mathrm{C}}_{\max }=0.25 \mathrm{at} \mathrm{r} / \mathrm{b}=\forall 0.6$ which dipped to 0.22 at the centreline. The axisymmetric plumes initially had the double peaked shape, which, further from the port, changed to a rounded bell shape with a peak value of 0.40 .

\section{Intermittency}

The intermittency of a flow is a measure of the temporal fluctuations in the position of the flow. The advected thermal is intermittent due to its turbulent nature and the irregular development of the sub-thermals. The intermittency, $\gamma$, quantifies the fraction of time that the flow can be expected at a given location. The presence of the flow is recognized when the concentration is greater than a threshold value, $c_{t}$, which is a fraction of the maximum of the averaged concentration profile. The intermittency is defined as,

$$
\gamma=\lim _{T \rightarrow \infty} \frac{1}{T} \int_{0}^{T} I(t) d t
$$

where $\mathrm{I}(\mathrm{t})=1$ if $\mathrm{c}(\mathrm{t}) \geq \mathrm{c}_{\mathrm{t}}$ and $\mathrm{I}(\mathrm{t})=0$ if $\mathrm{c}(\mathrm{t})<\mathrm{c}_{\mathrm{t}}$.

Some previous studies have looked at the intermittency of buoyant jet flow. Papps [9] looked at the intermittency of merging buoyant jets and found they were self- similar when scaled to the maximum concentration of the averaged profile for that profile.

The normalized instantaneous concentration profiles are analyzed according to equation 2 , using a threshold concentration scaled to the maximum concentration of the time averaged profile of $\mathrm{c}_{\mathrm{t}}=0.15 \overline{\mathrm{C}}_{\text {max }}$. This threshold value shows the most detail, without becoming highly erratic due to the resolution of the image at the threshold concentration. Papantoniou and List [8 ] found the analysis to be insensitive to values of $c_{t} / \overline{\mathbf{C}}_{\max }$ between 0.05 and 0.15 . The instantaneous intermittency profiles are averaged and this represents the fraction of time that the location is occupied by the flow, or equivalently, the probability of finding the flow at that location.

The normalised intermittency profiles along the trajectory are self-similar except for the profile closest to the port as, at this point, the subthermal structures are not yet established as seen in Fig.12. The profile is approximately Gaussian in shape, but offset horizontally by $0.3 \mathrm{r} / \mathrm{b}_{\mathrm{V}}$ towards the trailing edge and upwards by 0.09 (with the leading edge then approaching -0.09) and 
the peak of the profile is reduced asymmetrically with a greater reduction at the side of the leading edge.

The intermittency profiles do not reach a value of 1 at any point in the profile, which indicates that at some point in time there can be ambient fluid at any point in the flow region. This can be inferred from the 'broken up' and irregular nature of the flow, seen in Fig. 3 and Fig. 4, and by the minimum instantaneous concentrations of zero at any point in the profiles. This is in contrast to a jet, which has an intermittency value of 1 about the centreline indicating that jet fluid is always present at the centreline. The shift of the intermittency profile towards the trailing edge, as compared to the average profile, can be explained by the higher concentrations found towards the leading edge. These higher concentrations mean that the flow is present less often to average out to a given average concentration at the leading edge as compared to the trailing edge. An intermittency of between 0.05 and 0.15 extending away from the trailing edge at approximately $\mathrm{r} / \mathrm{b}_{\mathrm{V}}=-2$, again indicates the presence of buoyant fluid that is left behind by the main downwards advected thermal flow.

\section{Conclusions}

A visual inspection of the advected line thermal indicated that it was a complex and irregular flow, characterized by subthermal formation.

The investigation into the instantaneous character of the advected thermal up to $\mathrm{x} / \mathrm{l}_{\mathrm{PA}}=1000$ through statistical studies of the maximum and minimum of the instantaneous concentration profiles, the root mean squared concentration deviations and the intermittency of the flow reveal the variability in the flow. Maximum instantaneous concentrations of up to 3.5 to 4 times the maximum concentration of the averaged flow are found in profiles that are skewed towards the leading edge of the flow. Minimum instantaneous concentrations of zero are found throughout the flow. The $\mathrm{rms} \mathrm{c} / \overline{\mathrm{C}}_{\max }$ profiles have a maximum value of 0.8 which is found towards the leading edge. The shape of the $\mathrm{rms} \mathrm{c}^{\prime} / \overline{\mathrm{C}}_{\max }$ profile is roughly similar to that of the maximum instantaneous concentration profile. The maximum value of $\mathrm{rms} \mathrm{c}^{\prime} / \overline{\mathrm{C}}_{\max }$ for the advected thermal is greater than that for both the plume and jet because of the large scale variability in the flow caused by the subthermal structures. The intermittency of the flow is found to almost follow a shortened Gaussian profile that is offset towards the trailing edge of the flow as compared to the averaged concentrations.

These statistical studies indicate several details of the instantaneous structure. Intermittent but 
high concentrations occur towards the leading edge of the advected thermal and its subthermals. High concentration gradients at the leading edge are caused by these regions of high concentration at the leading edge. These regions of high concentrations lead the downward flow because of the greater buoyancy force driving their motion, as compared to the less concentrated regions. Small amounts of buoyant fluid do not get entrained into the main downward movement of the advected thermal and trail behind as a sort of wake. The study also illustrates the fluctuating and intermittent character of the advected thermal, which is due mainly to the turbulent development of the subthermal structures.

Acknowledgments The first author would like to acknowledge the support of a Commonwealth Scholarship during the research.

\section{Notation}

b radius at which the local velocity, $\mathrm{u}_{\mathrm{eg}}$, is $1 / \mathrm{e}$ of the maximum velocity of the Gaussian distribution, $\mathrm{U}_{\mathrm{eg}}$

$b_{\mathrm{V}} \quad$ Gaussian or time averaged concentration half-width in vertical direction

c local tracer concentration, subscript indicates instantaneous or averaged

c' local concentration fluctuation

$c_{t} \quad$ threshold concentration for intermittency determination

$\overline{\mathrm{C}}_{\max } \quad$ time averaged centreline tracer concentration

$\mathrm{d}_{\mathrm{p}} \quad$ port diameter

$\mathrm{Fr}_{0} \quad$ port densimetric Froude number $\left(\mathrm{U}_{0} /\left(\Delta_{0} \mathrm{~d}_{\mathrm{p}}\right)^{0.5}\right)$

$l_{\mathrm{P}, \mathrm{A}} \quad$ dimensionless length scale for the transition from a plume to an advected thermal $=\mathrm{q}_{\Delta 0} / \mathrm{U}_{\infty}{ }^{3}$

$\mathrm{q}_{\Delta 0} \quad$ initial buoyancy flux of jet $\left(\pi / 4 \mathrm{U}_{0} \mathrm{~d}^{2} \Delta_{0}\right)$

$\mathrm{r} \quad$ radial distance from axis

Re Reynolds number

$\mathrm{U}_{0}, \mathrm{U}_{\infty}$ initial velocity of jet at port exit, crossflow velocity

$\mathrm{x}, \mathrm{z} \quad$ horizontal distance from port parallel to jet, vertical distance from port

$\Delta_{0} \quad$ initial buoyancy $=\mathrm{g}\left(\rho_{0}-\rho_{\mathrm{a}}\right) / \rho_{\mathrm{a}}$

$\gamma \quad$ intermittency 
$\rho_{0}, \rho_{\mathrm{a}} \quad$ initial advected thermal density, ambient density

\section{References}

[1] Cresswell R.W., Morton, B.R., Nguyen K.C. and Baines D.G., (1992c), Thermals, puffs and mass sources 3 - laboratory studies, $11^{\text {th }}$ Australasian Fluid Mechanics Conference, 2: 11491152.

[2] Gaskin, S.J. (1996), Single buoyant jets in a crossflow and the advected line thermal, Ph.D. Thesis, University of Canterbury, Christchurch, New Zealand.

[3] Kotsovinos, N.E. (1975), A study of the entrainment and turbulence in a plane buoyant jet, Report No. KH-R-32, W.M Keck Laboratory of Hydraulics and Water Resources, California Institute of Technology, Pasadena, California, U.S.A.

[4] Lee, J.H.W. and Cheung, V., (1991), Generalized lagrangian model for buoyant jets in current, J. Environmental Engineering, ASCE, 116: 1085-1106.

[5] Morton, B.R., Cresswell R.W. and Nguyen K.C. (1992a), Thermals, puffs and mass sources 1-similarity and variability, $11^{\text {th }}$ Australasian Fluid Mechanics Conference, 2: 1141-1144.

[6] Nguyen K.C., Morton, B.R. and Cresswell R.W. (1992b), Thermals, puffs and mass sources 2 - numerical studies, $11^{\text {th }}$ Australasian Fluid Mechanics Conference, 2: 1145-1148.

[7] Papaniculaou, P.N., (1984), Mass and momentum transport in a turbulent buoyant vertical axisymmetric jet, PhD thesis, W.M. Keck Laboratory of Hydraulics and Water Resources, California Institute of Technology, Pasadena, California, U.S.A.

[8] Papantoniou, D. and List, E.J. (1989), Large scale structure in the far filed of buoyant jets, $J$. Fluid Mechanics, 209: 151-190.

[9] Papps, D.A. (1995), Merging buoyant jets in stationary and flowing ambient fluids, Ph.D. Thesis, University of Canterbury, Christchurch, New Zealand.

[10] Perry, A.E. and Lim, T.T. (1978), Coherent structures in coflowing jets and wakes, J. Fluid Mechanics, 88(3): 451-463.

[11] Richards, J.M. (1963), Experiments on the motions of isolated cylindrical thermals through unstratified surroundings, International Journal of Air \& Water Pollution, 7: 17-34.

[12] Scorer, R.S. (1957), Experiments on convection of isolated masses of buoyant fluid, J. Fluid Mechanics, 2: 583-594.

[13] Tsang, G. (1971), Laboratory study of line thermals, Atmospheric Environment, 5: 445-471.

[14] Wood, I.R., Bell, R.G. and Wilkinson, D.L. (1994), Ocean disposal of wastewater, World 
Scientific Publishing Co. Ltd., Singapore. 
Figures for "Flow structure and mixing dynamics of the advected line thermal"

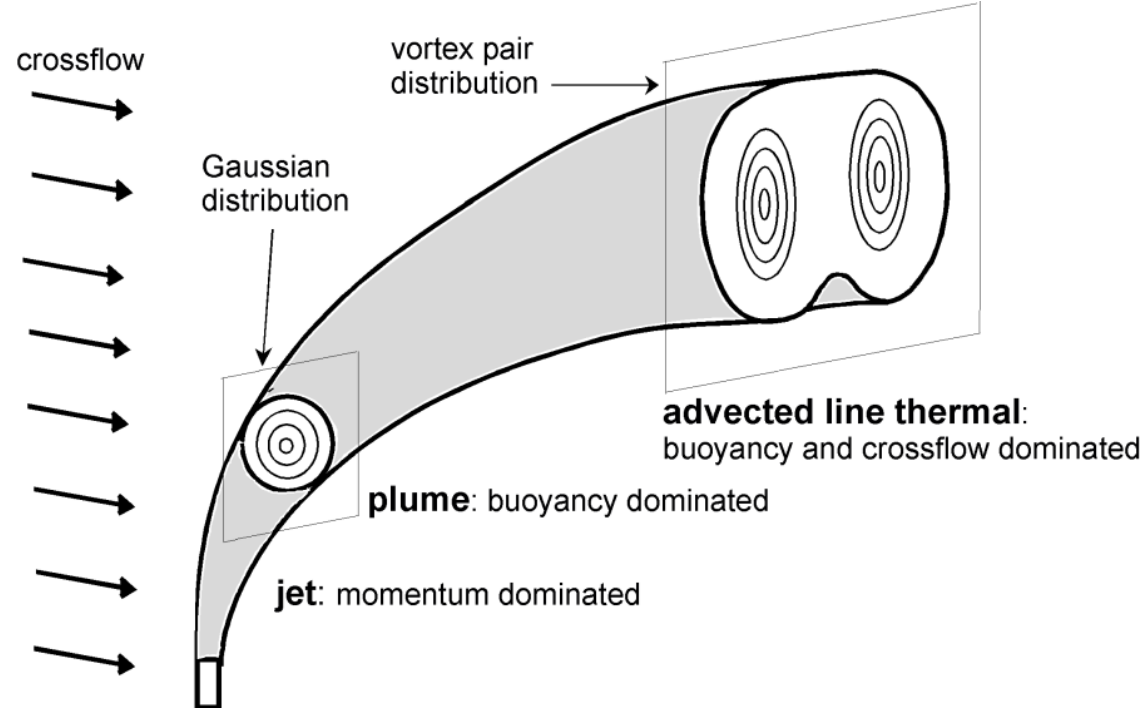

Fig.1. The flow asymptotic flow regions of a buoyant jet in a crossflow. 


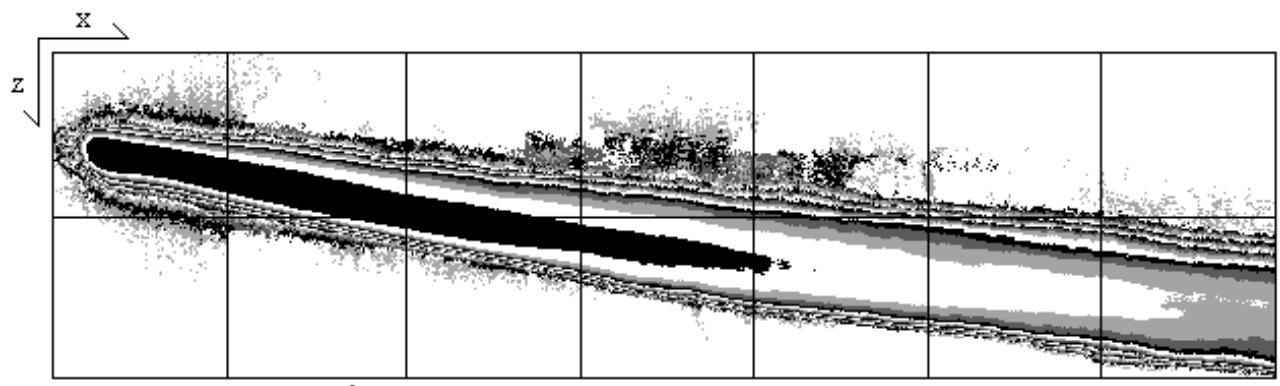

Grid at $100 \mathrm{~mm}, 65 / \mathrm{P}, \mathrm{A}$

$\mathrm{clCo}$

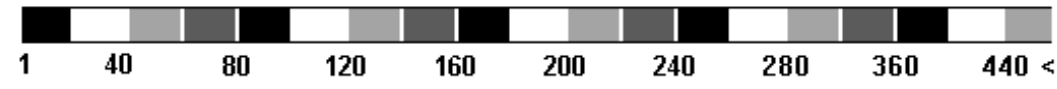

(a) Longitudinal centreline section, $4 \mathrm{~s}$ average
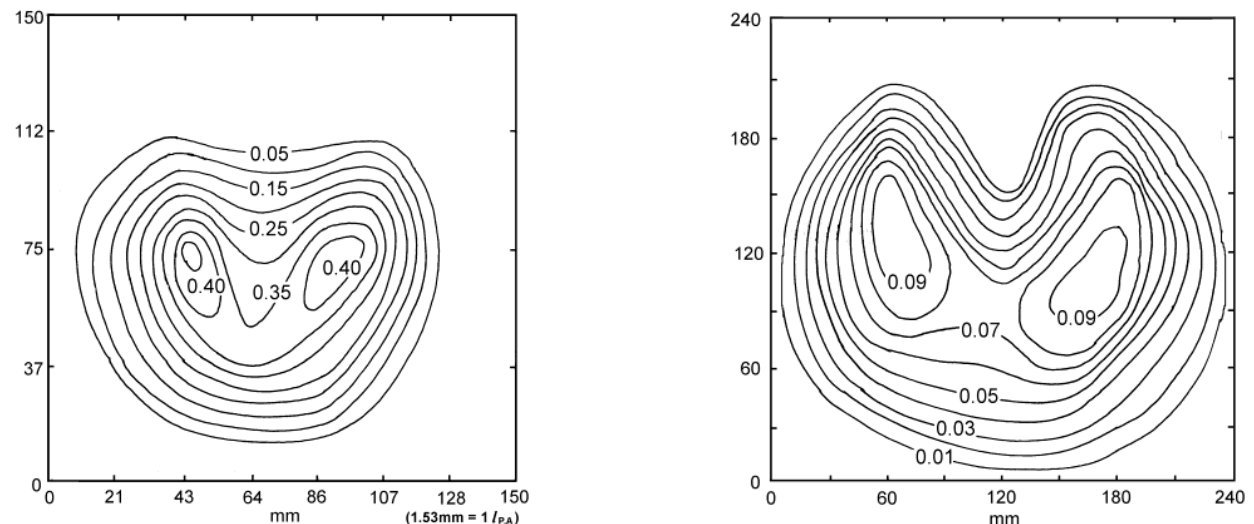

(b) Cross-sections at $\mathrm{x} / l_{\mathrm{P}, \mathrm{A}}=455, \mathrm{z} / l_{\mathrm{P}, \mathrm{A}}=65$ and at $\mathrm{x} / l_{\mathrm{P}, \mathrm{A}}=1300, \mathrm{z} / l_{\mathrm{P}, \mathrm{A}}=127,30 \mathrm{~s}$ average (concentration contours in $\mathrm{g} / \mathrm{l}$ salt)

Fig. 2. Time averaged structure of an advected line thermal. 

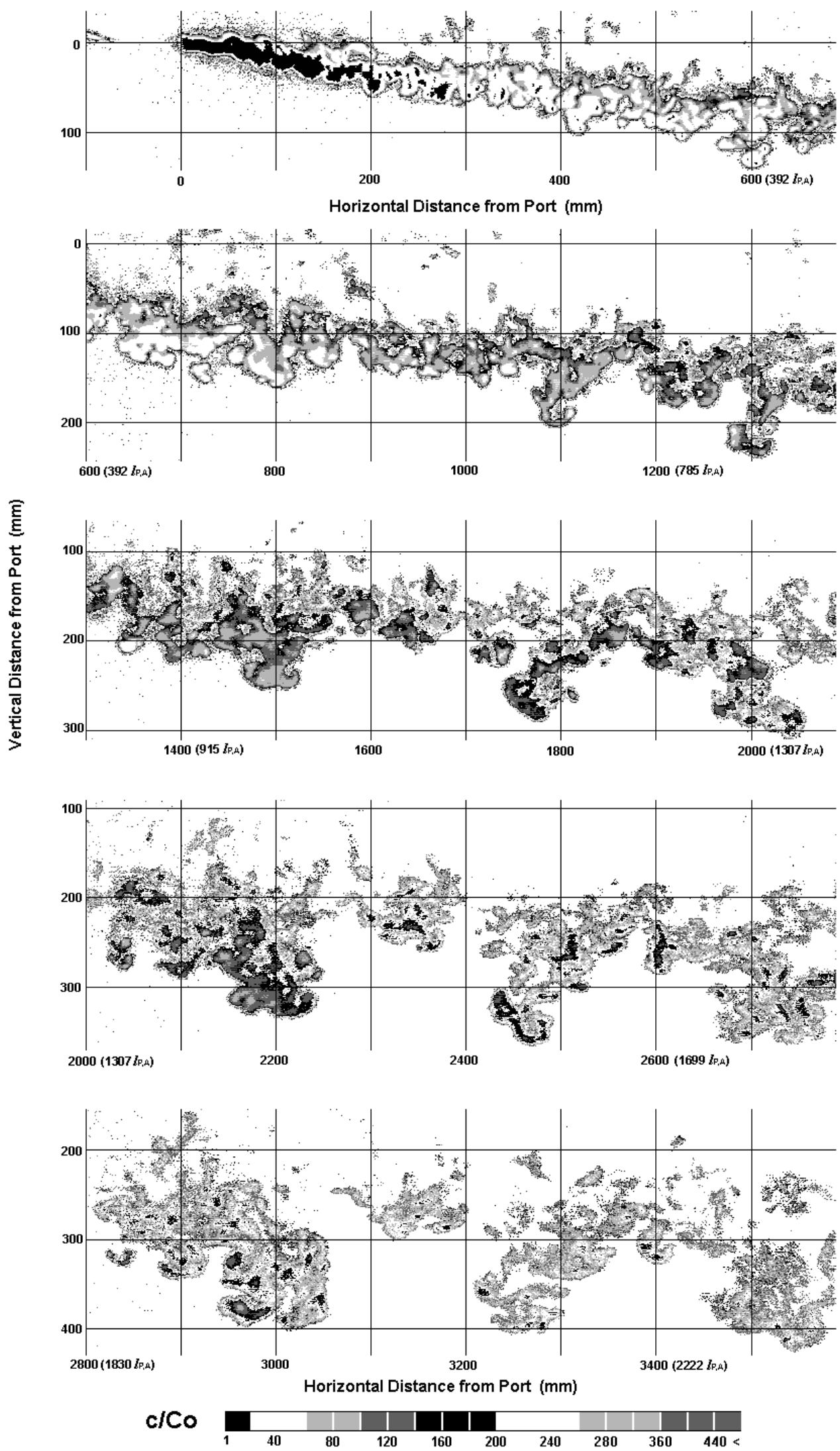

Fig. 3. Development of the advected line thermal seen from a longitudinal centreline section of the flow. 


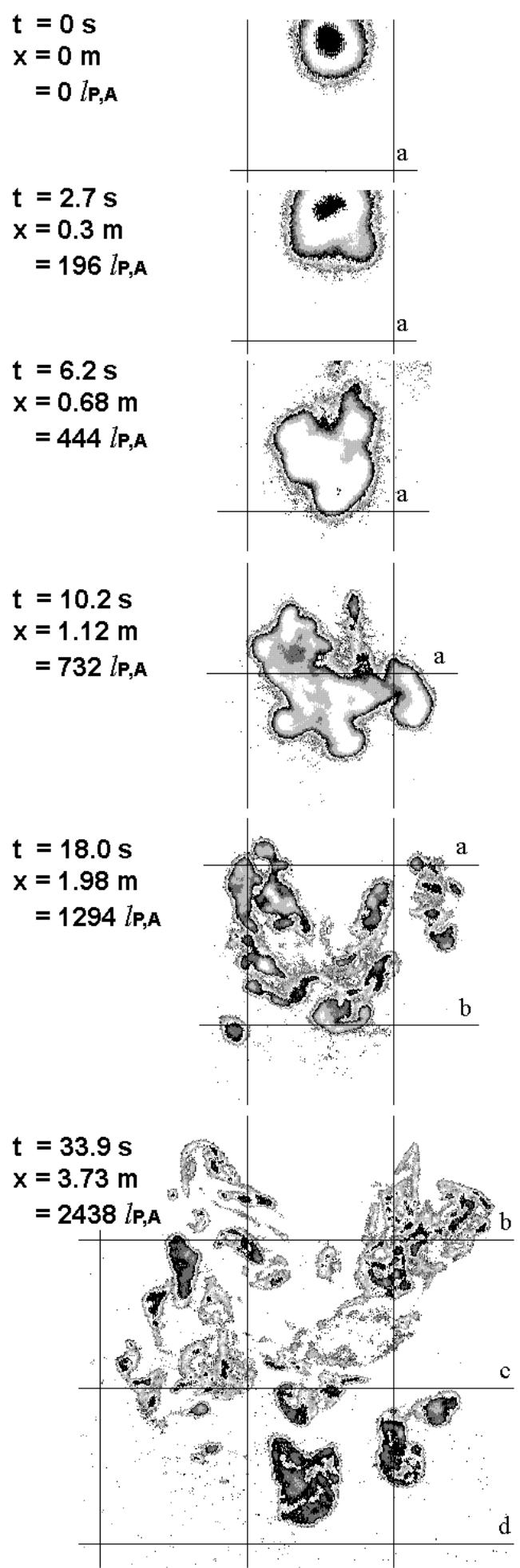

(a) Between subthermals

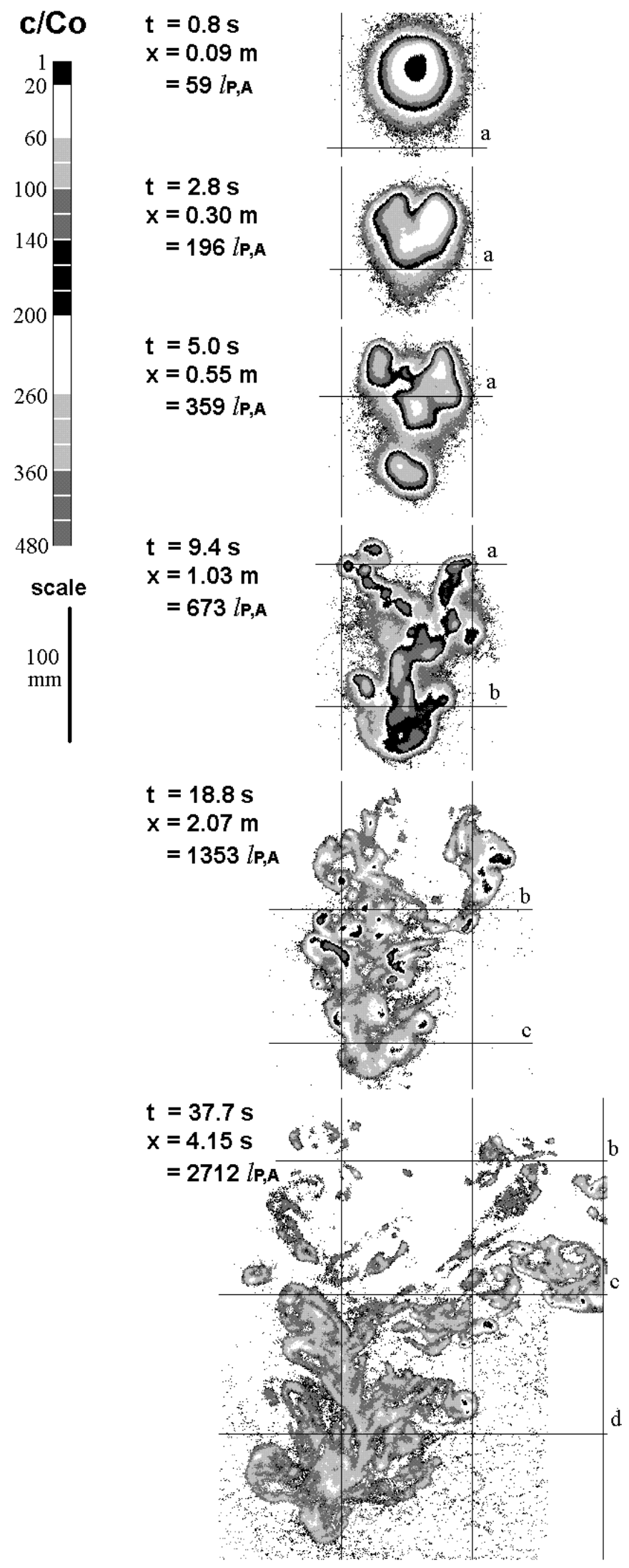

(b) At subthermals

Fig. 4. Development of the advected line thermal seen from a perpendicular cross-section of the flow. 


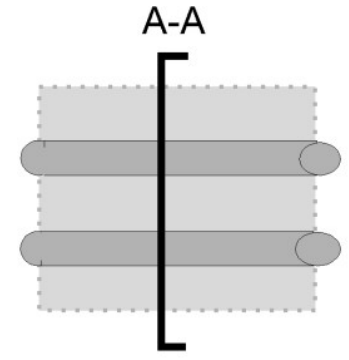

Top view: line thermal,

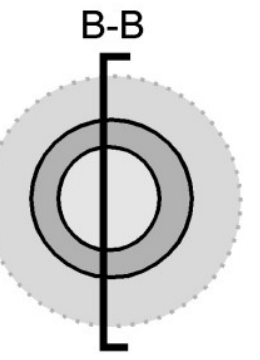

3-D thermal.

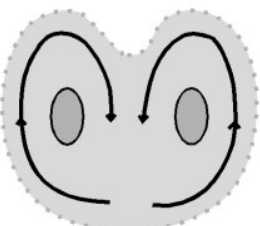

Section A-A and B-B

Fig.5. Sketch of a line thermal and a 3-dimensional thermal.

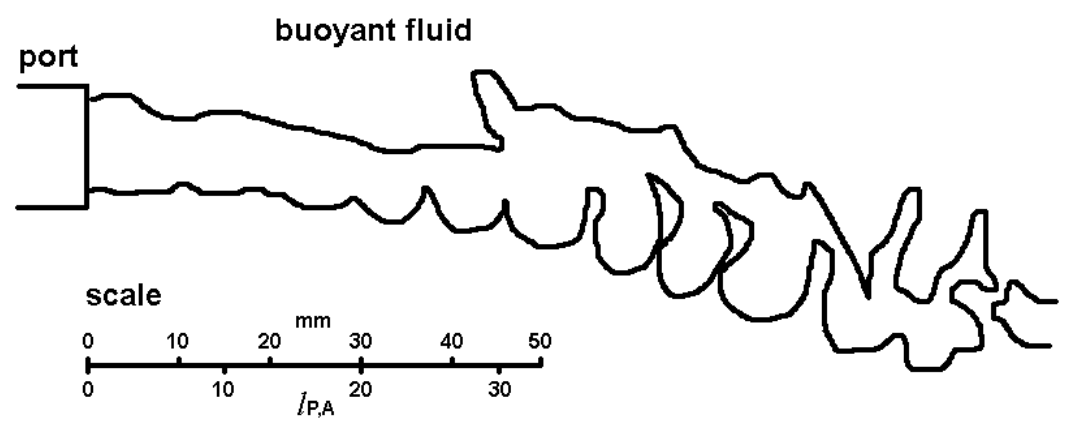

Fig.6. Structure of the advected line thermal flow close to the port exit.

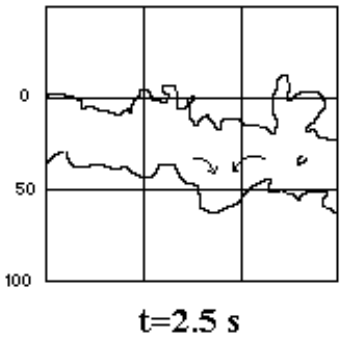

Grid at $50 \mathrm{~mm}, 33 l_{P, A}$ arrows indicate movement of buoyant fluid

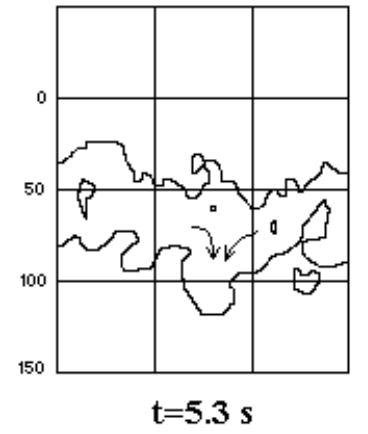

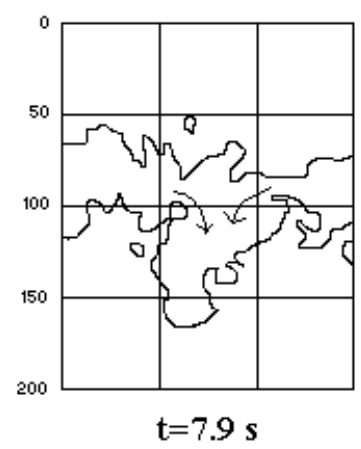

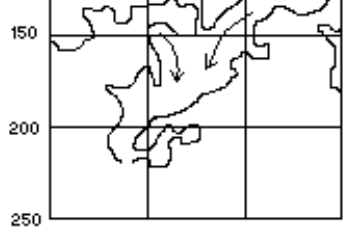

$\mathrm{t}=12.1 \mathrm{~s}$

Fig.7. Lateral movement of the buoyant fluid in the advected line thermal leading to the breaking up of the initial flow structure 

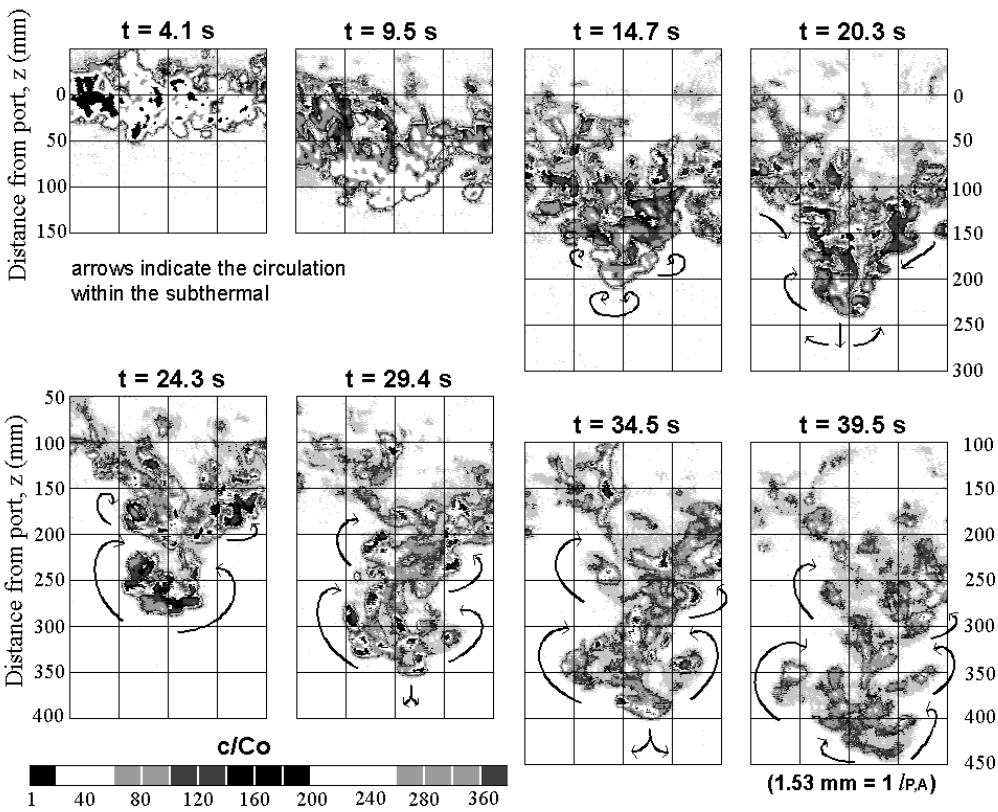

Fig. 9. Later stages of subthermal development showing successive cap and vortex pair structures.

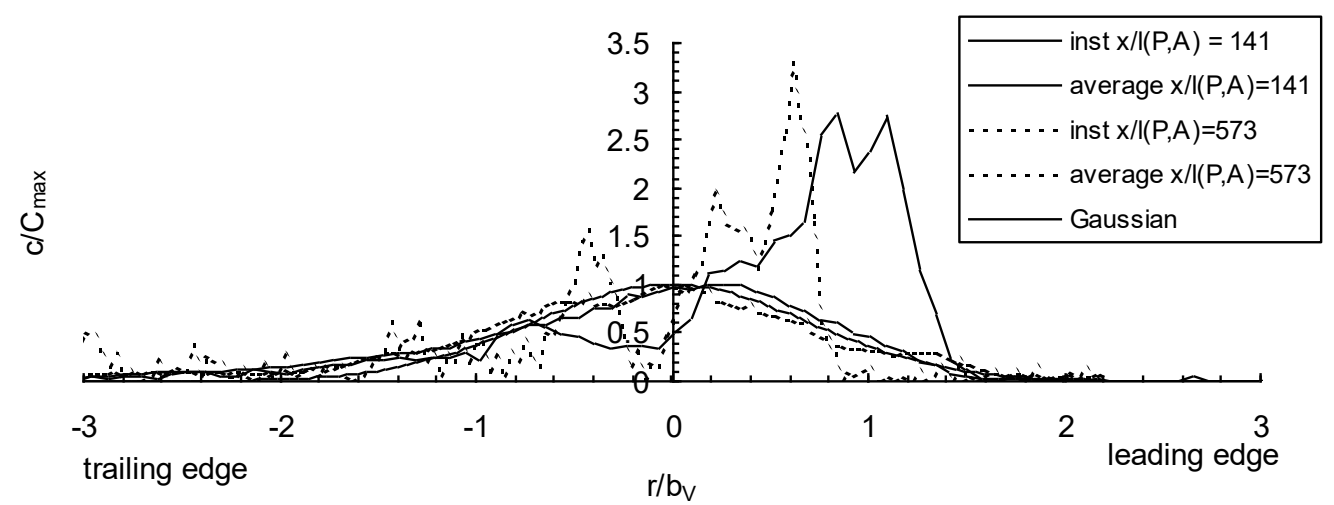

Fig. 10. Profile of the instantaneous concentration (chosen at a subthermal location) and the average concentration of the advected line thermal compared to the Gaussian shape. 


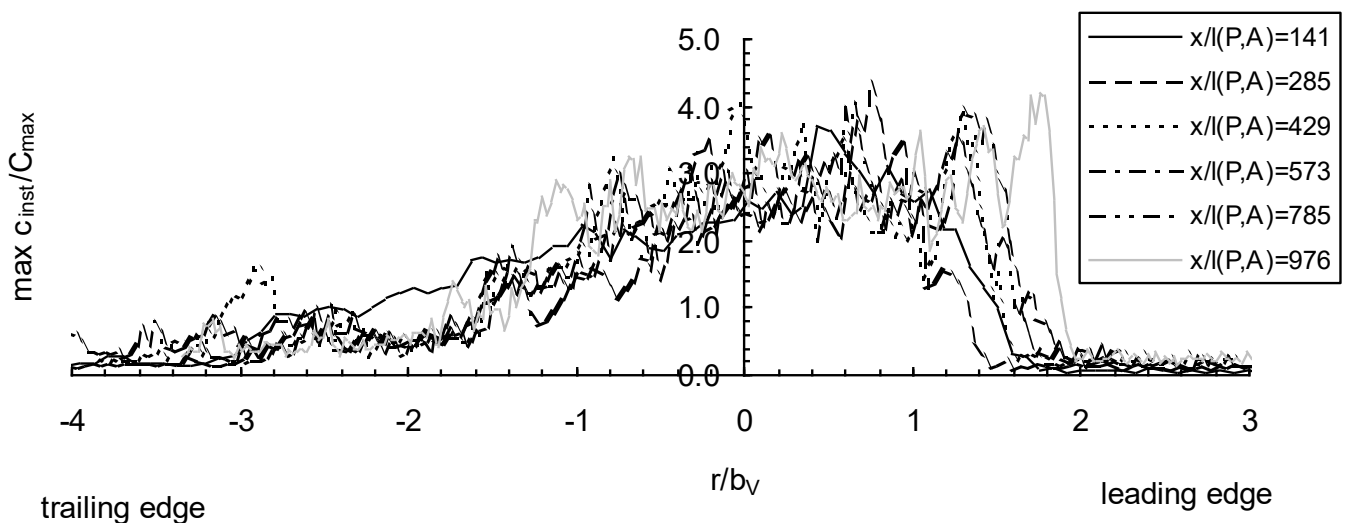

(a)

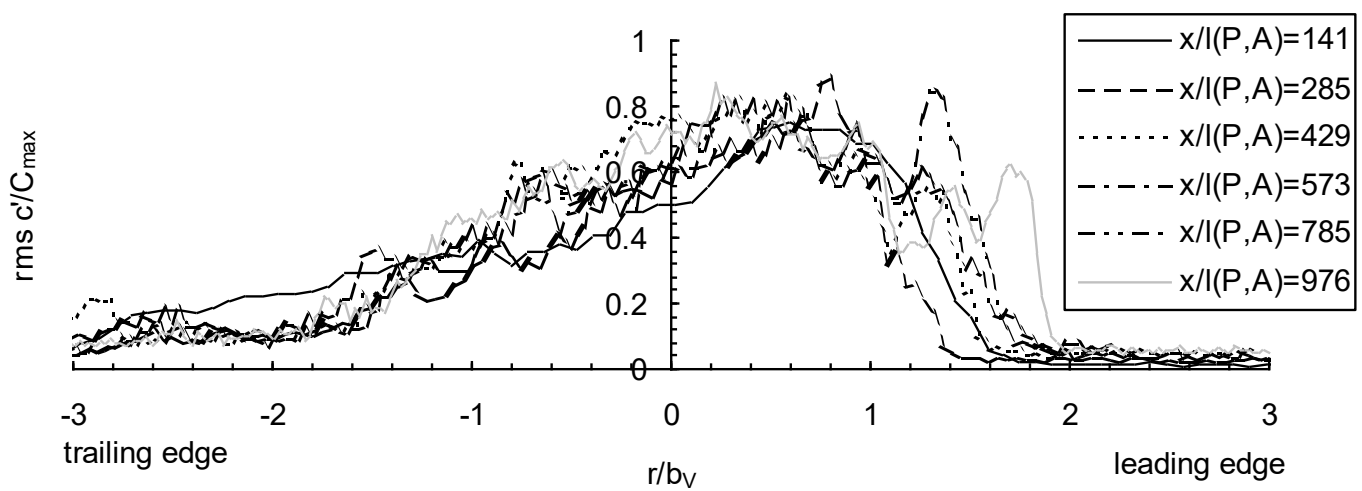

(b)

Fig. 11. Profiles of the advected line thermal of (a) maximum instantaneous concentration, (b) root mean squared concentration fluctuation 


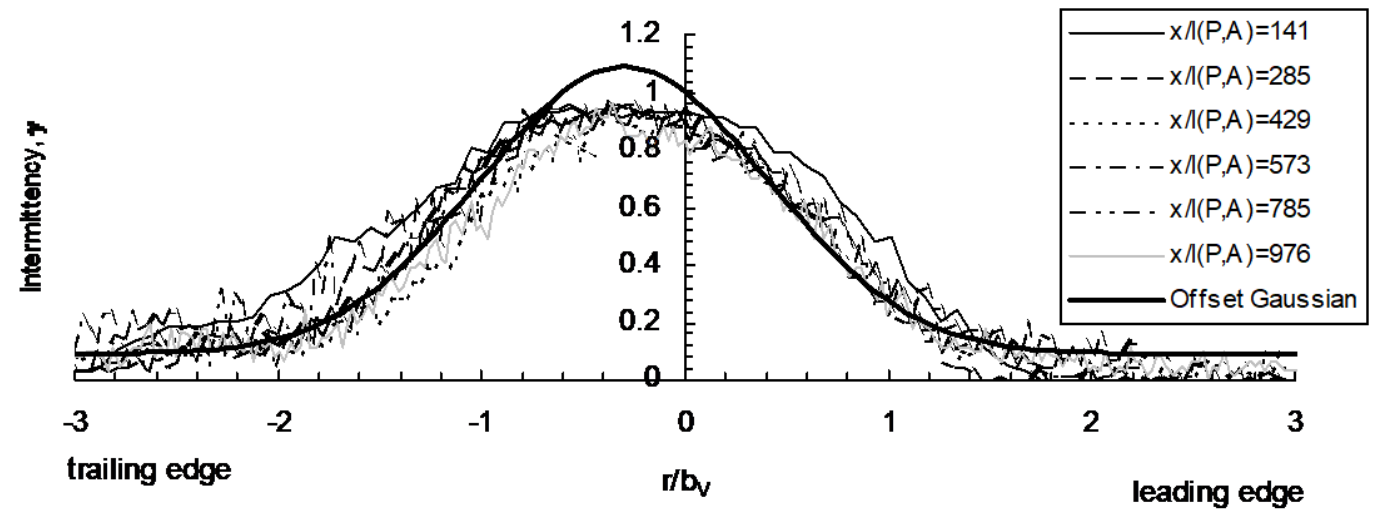

Fig. 12. Intermittency of the advected line thermal. 